\title{
Lazzaro e la riscrittura pirandelliana del mito biblico
}

Scritto o perlomeno già compiutamente ideato fin dal 1926 - quindi parallelamente alla Nuova colonia, prima opera della trilogia mitica ${ }^{1}$ - il Lazzaro fu poi compiuto nell'estate del 1928, quando in soggiorno a Viareggio Pirandello ne fece la prima lettura ad un gruppo di amici. Andò poi in scena il 9 luglio 1929 al Royal Theatre di Huddersfield, nella versione inglese di C.K. Scott-Moncrieff, e fu infine portato nei teatri italiani dalla Compagnia di Marta Abba solo nel dicembre di quello stesso anno, come era successo anche con altri drammi pirandelliani, che in quegli stessi anni erano stati rappresentati prima all'estero che in Italia. $^{2}$

Il mito pirandelliano fin dal suo primo apparire causò accese polemiche negli ambienti cattolici, che lo accusarono di demolire le verità fondamentali dell'educazione cattolica e della fede cristiana (Giudice 529-30). Lo scrittore, da parte sua, aveva precisato fin dall'inizio nell'intervista rilasciata a Romano Drioli che, se il suo intento era senza dubbio religioso, per concretizzarlo in forma artistica e per renderlo valido all'uomo d'oggi, aveva dovuto trasformare l'evento biblico attingendo a modelli psichici universali di chiara matrice archetipica:

Ho scritto [. . .] un Lazzaro moderno . . . . Nulla è fissato storicamente. Perciò non potevo attingere al Lazzaro del mito ebraico, ma a un Lazzaro dei nostri giorni. [. . . ] Ma come fu nel mito ebraico, il problema religioso di Lazzaro può ripresentarsi sempre alla coscienza degli uomini. [. . . ] Un moderno non può che superare il trascendentalismo ed è perciò che infatti io ritomo al mito religioso, ma attraverso l'immanenza dello spirito.

L'insistenza, appunto, sul fatto che "il problema religioso di Lazzaro può ripresentarsi sempre alla coscienza degli uomini" (Drioli), ritomerà poi anche nella Premessa alla prima edizione (1929):

Non è il Lazzaro biblico. È il mito di Lazzaro in ciò che ha di eterno: il valore spirituale, supremo della resurrezione per cui può essere di ieri come di oggi. È un Lazzaro di oggi. (Massi 8)

Visto in questa luce, è chiaro che nel processo di rigenerazione che dall'individuo passa poi alla società l'esperienza religiosa di questo tipo è la prima a cui si va incontro e questo spiegherebbe perché, nonostante La nuova colonia fosse stata scritta e rappresentata prima del Lazzaro, Pirandello invertì in un secondo 
tempo l'ordine dei primi due miti in una Nota apparsa su Nuova Antologia il 16 dicembre 1931. In tale occasione, con la pubblicazione concomitante del primo atto dei Giganti della Montagna dal titolo I fantasmi, Pirandello forniva una prima sistemazione alla sua trilogia mitica che, trascurando l'ordine cronologico di composizione delle opere, le elencava con il Lazzaro come punto di partenza del proprio discorso mitico e come recupero di quella matrice archetipica del mito ebraico su cui innestare la propria riscrittura.

\section{Campagna e città e la dicotomia di vita e non-vita}

L'opposizione antifrastica tra campagna e città - topos che ricorre come un vero leitmotiv in tutta l'opera pirandelliana e che già nella Nuova colonia si configurava nel contrasto tra isola e porto di mare - nel Lazzaro è portata avanti da vari personaggi anche minori, quali per esempio Deodata la governante, ma riceve la sua espressione più compiuta nelle parole di Sara:

Di qua si vede la città — non potei più guardarla [. . . ]. L'odio di quelle chiese, di quelle case, e il tribunale . . . tutto! [ . .] Buttai via tutto e mi feci contadina contadina qua, sotto il sole, all'aperto! [ . . .] quest'uomo [Arcadipane . . . ] impedì che mi dannassi, insegnandomi le cose della campagna, la vita, la vera vita che ha qui, fuori della città maledetta, la terra. (1198-99)

Dove alla luce dello spazio aperto, che consente a mente e corpo di espandersi e fiorire, si contrappone il senso di chiusura e di oppressione della "città maledetta", epitome con le sue chiese, le sue case ed i suoi edifici pubblici, primo fra tutti il tribunale, della società organizzata secondo leggi che controllano e opprimono. Si noti come la città assuma per Sara il significato di simbolo del potere dei Padri. Nel discorso di Sara, infatti, le chiese stanno per la religione istituzionalizzata piuttosto che essere simboli di fede, mentre le case e il tribunale si prefigurano essenzialmente come la sede del potere patriarcale nella famiglia e nelle leggi. La città per Sara s'identifica, quindi, all'interno della diade campagna-città con il regno dei Padri, percepito come fondamentalmente ostile al Femminile. Alla luce della percezione del personaggio - che è portatore del messaggio di fondo - la città significa, dunque, un totale snaturamento dei valori più autentici della vita $\mathrm{e}$, per chi vi vive, uno stato di esilio dalla vita.

$\mathrm{La}$ "vita vera" è, invece, quella della terra: il "crescere", il "fiorire" e il "fruttare" e, come dice il personaggio: "la gioja della pioggia che viene a tempo; e l'afflizione della nebbia che brucia gli olivi sul fiorire" (1199). La terra, quindi, è percepita come natura provvida, come Madre fertile e dispensatrice di bene e di nutrimento. Sul podere, microcosmo che però concentra e riassume la totalità dell'universo, viene proiettato l'archetipo della Natura come Terra Madre. Qui il tempo storico è sospeso e prevalgono lo spazio e la temporalità mitici: cessato di esistere il sociale inteso come sovrastruttura, ciò che conta sono solo più l'Uomo, la Donna, i Figli e la Terra uniti in una condizione di participation mystique, non più individui, ma tipi umani, la cui unica differenziazione è il ruolo che svolgono 
in quel contesto di primigenia naturalità. Non esistono tensioni né conflitti in questo stato di armonia ed equilibrio, come spiega Sara di sé: "[ . . ] non desidero perché ho; non spero perché, ciò che ho, mi basta; ho la salute, il cuore in pace e la mente serena" (1199).

Il podere finisce con il configurarsi, quindi, come paradiso terrestre, luogo e condizione di unità primordiale con il Tutto, sogno di totalità, nostalgia dello stato indifferenziato, uroborico della coscienza. Nel giardino primordiale vivono in perfetta armonia l'uomo buono e puro e la donna sana e fertile, e la terra è cornucopia. Qui zampilla il ruscello o la fonte della bevanda sacra legata ai simboli del rinnovamento ciclico (Durand 260). È emblematico che anche nel podere l'acqua affiori dal sottosuolo e lo trasformi interamente, come spiega Sara a Diego:

l'orto, la vigna, il frutteto: uh, frutta per tutte le voglie! Abbiamo trovato l'acqua, sai? quella vena che dicevi tu, che certe volte, ricordi?, si sentiva scorrere sotto il ciglio del sentiero che conduce alla vallata: quella! Una ricchezza. Ha rinfrescato e rinnovato tutto. Tre vivai grandi sempre pieni, e scorre per le zane, da per tutto, allegra, e ti fa tirare dal fondo dei polmoni il respiro quando ne senti il fragore, certe sere di caldo. (1180)

Quell'acqua che sgorga e tutto nutre di sé è il corrispettivo simbolico della vita che sgorga dagli strati profondi della psiche, la fonte di ogni rinnovamento della vita. In questo senso inconscio e madre coincidono, perché entrambi sono i principi generatori rispettivamente della vita psichica e di quella biologica. Questo spiega anche perché il senso di pienezza e di totalità del giardino archetipico sia direttamente collegato al Femminile nella sua doppia valenza di generazione e di trasformazione. È quindi chiaramente emblematico che nel Lazzaro la scoperta dell'acqua e la fertilità prorompente del podere coincidano con la venuta di Sara e con la sua maternità.

Nel contempo il podere si definisce sempre più chiaramente anche come lo spazio archetipico privilegiato della trasformazione. Non è un caso che in tale spazio mitico avvengano tutte le vere rinascite dell'opera: qui Sara trova se stessa ed il più autentico senso della vita, qui viene Lucio a "rinascere in lei" e a trovare il senso della propria missione e della propria fede, qui Diego muore a se stesso ed alla propria fede astratta per trovare il germe di una nuova vita spirituale, qui Lia ritorna alla pienezza di vita riacquistando l'uso delle gambe. La città, quale luogo in cui Diego era morto (prima metaforicamente nello spirito e poi anche nel corpo), la famiglia Spina si era totalmente disgregata e la missione sacerdotale di Lucio era entrata in crisi e finita, si definisce sempre più chiaramente nel procedere degli eventi come un principio di morte e di annientamento, mentre il podere invece si contrappone ad essa come l'Eden archetipico di nascita e di rigenerazione, come il Paradiso, simbolo del divenire del Sé e del processo di individuazione. ${ }^{3}$

La figura della Madre al suo centro, assume, allora, il significato della totalità 
e diventa portatrice del senso più profondo e più agognato di redenzione. Sullo hieròs gámos di Uomo e Donna primordiali, che viene adombrato nell'opera dall'unione di Sara ed Arcadipane, s'incentra così il mito della rinascita.

\section{Sara ovvero la Donna-Natura}

Sara è la Donna-Natura che ha gettato la maschera dei ruoli e delle convenzioni sociali. È la donna che per odio ha distrutto (una casa, una famiglia, uno stato sociale), ma che per amore e con amore è anche rinata alla vita. Odio e amore, vita e non essere sono le due polarità del personaggio, che ha ripudiato e distrutto per poter riaccogliere, ricostruire ma, questa volta, secondo principi che rispettino le leggi naturali e divine. È proprio questo suo essere una forza di natura, senza conflitto, pur nella sua ambivalenza, e senza 'coscienza', che ne fa una perfetta manifestazione archetipica. E Pirandello la dipinge, infatti, al suo primo presentarsi in scena con tutta la numinosità di un'apparizione divina: la sua bellezza è 'ineffabile', perché sovrumana supera i limiti del dicibile, ed è 'nuova' perché sempre rinnovantesi, 'sana' perché il divino è integro, non ha difetti, e 'potente' perché possiede tutta la forza e l'energia che distinguono appunto l'essenza divina dall'umana fragilità.

La condizione di Sara prima di abbandonare Diego e di rifugiarsi nel podere, invece, era quella di esiliata dalla vita. Diego, grazie al suo potere di capofamiglia, le aveva letteralmente sottratto i figli per allevarli in istituti religiosi secondo i suoi principi, ma così anche svuotando la casa e privando Sara del privilegio fondamentale per una madre di prendersi cura dei propri figli. E la madre in Sara si ribella:

Mi si torcevano le viscere, vedendo così - l'uno e l'altra - teneri teneri - avvizzire; e vedendo lui, vostro padre [. . .] duro, ostinato per non darmela vinta. [. . .] Sentivo che non era vita per me da potersi reggere, con questo scempio che vedevo fare di voi, come alla mia stessa carne. (1197)

Ma la ribellione di Sara deve fare i conti con la mentalità patriarcale della società in cui vive, che riconosce sopra ogni cosa solo la patria potestà e vede la donna come una pura appendice del marito. Il tribunale, a cui s'appella per veder riconosciuto anche il suo diritto di madre, in quanto garante della legge dei Padri non può che darle torto, come spiega al figlio Lucio:

[. . .] dissero che dovevo stare con lui e la figlia; e che la pretesa di levar te dal seminario non era giusta; e insomma che ero io - io e non lui - a voler la fine della famiglia. [... Q Quando a una madre si nega d'attendere ai suoi figli, a una madre che vuole la salute per i suoi figli le si dà torto che vuoi? ci si danna! (1198-99)

L'ostilità nei confronti del corpo che è propria della maschilità superiore ${ }^{4} \mathrm{si}$ manifesta in Diego verso Sara nel suo ruolo sia di madre che anche di moglie. Dirà, infatti, parlando dell'amore di Sara per i figli: "[. . .] li amava, d'un amore . .. non so, troppo carnale, a mio giudizio. Come tante madri, del resto" (1175). 
Questa carnalita, che appare costantemente nei discorsi di Sara a Diego, connota il personaggio di Sara nella sua totalità di donna. Intorno a tale fisicità si muove anche il rapporto tra lei e Diego, che è ambiguo perché pieno di contraddizioni da parte del marito. Diego, che non nega la forte attrazione erotica esercitata su di lui da Sara, nella sua tensione verso le cose dello spirito ne ha contemporaneamente timore e vergogna. L'Eros di Sara, che troverà poi piena espressione nella dimensione solare e naturale del podere e nell'amore semplice ma diretto di Arcadipane, non trova riscontro nelle zone d'ombra, piene di sensi di colpa di Diego. La sua "timidità" le ripugna perché la percepisce come malata ed insincera:

[. . . ] faceva il santo, il tiranno - ma poi, quello che più m'inferociva di lui, quando mi s'accostava, era quella mollezza della sua timidità . . . .

Tronca con un'esclamazione e un atto di schifo:

- ah Dio! - Eppure ti giuro, Lucio, avrei, avrei fatto il sacrificio di resistere all'orrore che ormai avevo, purché ne fosse venuto un bene a voi, a voi, figli. (1198)

L'effetto castrante della spiritualità patriarcale ${ }^{5}$ viene percepito e rifiutato da Sara, che archetipicamente cerca un rapporto di unione personale con il maschile, non solo come "il portatore di uno strumento fallico fecondante ma anche [come] una potenza spirituale, un eroe”. Infatti, spiega sempre Neumann (Storia 181-82), "il femminile si aspetta forza, intelligenza, impegno, coraggio, protezione e disponibilità a combattere. [Vuole la capacità di] . . . abbattere la barriera di spine e di fuoco che rappresenta l'inibizione e l'angoscia, sbloccare o risvegliare la femminilita, vincere una battaglia di intelligenza risolvendo enigmi, sconfiggere la depressione o la tristezza". Questi aspetti del carattere virile, che sono in gran parte soppressi o indirizzati in senso esclusivamente spirituale in Diego, saranno invece quelli che in Arcadipane salveranno ed indirizzeranno Sara verso la piena realizzazione di sé. Con la perdita dei figli era stata la natura orgiastica di Sara ad affiorare e ad avere il sopravvento:

[...] le suore [. . .], dopo avermela ridotta in quello stato [Lia], me la dovevano assistere e curare ... - [. . .] loro capisci? non io! - loro! - m'avventai come una belva contro una; [. . .] mi presero per indemoniata. [. . .] me ne fecero scappare scappare - come una pazza! [. . . Un bisogno mi prese, un bisogno d'essere selvaggia; un bisogno di cadere a terra la sera come una bestia morta sotto la fatica - zappando, pestando le spighe sull'aja con le mule, a piedi nudi, sotto la canicola, girando a tondo con le gambe insanguinate e gridando come un'ubbriaca - bisogno d'essere brutale con chi mi pregava che avessi pietà di me [. . . ] - quest'uomo puro puro, Lucio, come una creatura uscita ora dalle mani di Dio - quest'uomo che [. . .] impedì che mi dannassi, insegnandomi le cose della campagna, la vera vita [. . . ] che ora sento, perché le mie mani la servono [la terra], l'ajutano a crescere, a fiorire, a fruttare. (1198-99)

Il paradiso, in cui la terra si è trasformata riflettendo simbolicamente l'unione armonica degli opposti, può solo esser tale quando la natura orgiastica e poten- 
zialmente distruttiva della Madre-Natura trova compensazione nel principio ordinatore del Logos. Allora anche il suo carattere benefico, demetrico può manifestarsi come abbondanza di messi e fertilità.

Se ci si sofferma sulle parole-chiave che costituiscono i nuclei di significato delle battute di Sara nel primo atto, vediamo che, prese nel loro insieme, delimitano appunto un microcosmo esterno ed interiore ben definito: terra, orto, vigna, frutteto, frutta, acqua, vena, ricchezza, rinfrescato, rinnovato, vivai / sangue, figli, salute, letizia, rinascere, madre, ribenedetta (1180-82). Il microcosmo qui delimitato è quello della Natura, la cui fecondità è però assecondata ed imbrigliata dall'uomo. Il binomio uomo-natura è visto nei suoi aspetti positivi di simbiosi armonica e felice. In questo paradiso terrestre Sara e Arcadipane sono i novelli Adamo ed Eva prima della caduta, uniti in uno hieròs gámos di mitica memoria, fuori dalle leggi del tempo e della società umana.

Sara s'identifica in pieno con il carattere demetrico della terra, nutrita dallo stesso principio vitale che fluisce nelle vene della terra, lei stessa feconda e nuovamente madre. La terra che prende a fruttificare nel podere si correla, quindi, perfettamente al ritorno della maternità di Sara; motivo questo, che ricorre innumerevoli volte, pur con varie modifiche o sfumature diverse, nell'opera pirandelliana, e che identifica inequivocabilmente la Terra con il carattere primigenio e più autentico della donna, e quest'ultima con l'archetipo della Grande Madre, "guardiana in prima istanza dell'interiorità e dei territori profondi dell'istintualità, delle emozioni, del sentimento, cioè di quella che costituisce per Jung [. . . ] l'anima in senso lato" (Pulcini 189).

In effetti entrambi i motivi mitologici, biblico-cristiano e greco-pagano, confluiscono nel tema demetrico. Come i progenitori dell'umanità, così Sara e Arcadipane vivono sani e felici, benedetti dall'amenità del luogo e dall'abbondanza che il loro lavoro produce. Arcadipane è come il primo uomo, appena uscito dalle mani del Creatore, doveva essere, l'uomo di cui nella Bibbia si dice: "Il signore Iddio prese dunque l'uomo e lo pose nel giardino dell'Eden, perché lo coltivasse e lo custodisse", l'uomo giusto e puro in intimo contatto con la natura, da cui trae equilibrio e saggezza. L'Amore in senso lato è il suo leitmotiv, che si manifesta nella devozione con cui si prende cura di Sara, dei figli, della terra, nel rispetto sincero e senza pretesa di ricompensa della legge divina. Così come ha accudito, "servito" e fatto fruttificare la terra, altsettanto ha fatto con Sara, di cui ha indirizzato positivamente l'energia altrimenti caotica e distruttiva. In questo senso il 'servo' della terra è anche, con piena coerenza, il servo di Sara nella sua identificazione con la Terra Madre come lei stessa precisa a proposito del suo rapporto con lui: "[. . .] quest'uomo che non ha saputo mai tollerare che mi facessi uguale a lui" (1199), che non è soltanto una notazione di carattere sociale, ma anche un aspetto fondamentale del contesto mitico qui ricreato da Pirandello.

Arcadipane rappresenta, dunque, il Logos maschile che stabilisce il principio di ordine nel caos primordiale dell'Eros femminile, che a sua volta lo trasforma, ${ }^{6}$ e il giardino coltivato accudito e pianificato si fa metafora, allora, della capacità 
dell'uomo di indirizzare la Natura, e della possibilità di un'integrazione della mente e dell'opera umane nella prorompente vitalità della Natura stessa. E poiché le leggi che governano tale rapporto d'integrazione non sono dell'uomo ma della natura, esse di necessità lo trascendono e gli sono superiori. Da qui nasce il senso di sacralità del lavorare la terra, secondo un rito che, pur con l'avanzamento tecnologico, non è mai veramente cambiato e che pone l'uomo a diretto contatto con il mistero stesso della vita. È a questo senso del sacro che si rifa Sara quando parla del suo lavoro nei campi e della gioia della ricompensa dopo la fatica: "[. . .] e il piacere, il piacere, sai, di fare il pane con le tue stesse mani che hanno seminato il grano..." (1199). ${ }^{7}$

Alla luce di quanto si è venuto discutendo finora sul significato simbolico e sulle valenze archetipiche del podere e dei suoi coltivatori, il mito pirandelliano appare sempre più come la risposta alla storia biblica dei progenitori dell'umanità, ma nella direzione opposta. Sara è una Eva che, dopo essere stata esiliata sulla terra, ritorna al paradiso terrestre per riscoprime i più autentici valori, non più collettivi ma transpersonali, attuando cioè un recupero di ciò che, per sopravvivere come entità sociale, era andato perso irrimediabilmente. Si noti come questo aspetto del testo pirandelliano collimi perfettamente con l'analisi che della psiche moderna ha fatto Neumann (Storia 350): "Con l'abbandono del paradiso terrestre viene abbandonata anche la voce di Dio che parlava in esso, e affinché avvenga l'adattamento sociale diventa indispensabile assumere come valori dominanti i valori del collettivo, dei padri, delle leggi, della coscienza morale, dell'etica comune, ecc.". "La voce di Dio che parlava in esso" è appunto quel Dio immanente che scoprirà Lucio uscendo dalla sua crisi spirituale e di cui parla Sara a Diego: "(indica il Crocefisso). Tu non vedi che quello, e a tuo modo soltanto! [ . . . Sono la prima a inginocchiarmi! Ma Quello, sai, è lì per dare la vita, non per dare la morte!" (1181).

E Sara, anche in questo diametralmente opposta a Eva, sarà portatrice di vita e strumento di rinascita. Il Lazzaro si pone, quindi, come una riscrittura antifrastica del mito biblico della Genesi al cui centro, non più come ragione di onta e di dolore ma piuttosto come fonte di speranza e di vita, sta la figura della Madre.

\section{La liberazione dell'Anima ${ }^{8}$}

Il viaggio verso l'integrazione del materiale inconscio nella coscienza, che porta alla manifestazione del Sé, nel Lazzaro assume le vesti tematiche della ricerca di fede e del senso ultimo della vita e della morte. La ricerca di fede di Diego, tuttavia, per quanto sincera e sofferta, si è arenata nell'astrattezza, che l'ha privata del fervore e del calore che, soli, la possono far lievitare e trasformare in vita. L'istinto, le emozioni, l'interiorità sono tutte cose da cui rifugge, perché sfuggono al suo tentativo di controllare la vita, di organizzarla in chiare categorie a cui appellarsi nel bisogno e da cui sentirsi protetto e sicuro. Le leggi umane e perfino quelle divine ricadono in queste categorie, rispettano quella che sembra essere la logica della vita, almeno come la intende l'uomo-Diego. Dio allora è l'essere 
supremo, lontano e irraggiungibile, giudice impassibile delle umane debolezze e degli umani eroismi. Questa lontananza di Dio lascia l'uomo solo e sgomento a cercare la propria via di salvezza. Per Diego Spina questa via è fatta di dolore, di rinuncia, di dura negazione di sé; a questa non vita terrena ci sarà però la ricompensa di una vita nell'aldilà, come dichiara a Sara: "Che vuoi parlare tu di vita e di morte? Ti sei dimenticata che la vita vera è di là! Quand’è finita la carne ..." (1181).

I principi morali e religiosi per cui vive, pertanto, più che elevati sono astratti, cioè 'tratti fuori' dalla vita e proiettati fuori di sé, in un mondo di idee e di atti volontaristici, in cui non c'è posto per gli aspetti terreni dell'essere, né per le sue debolezze umane. Gli istinti e finanche gli impulsi più legittimi e naturali, quando non perseguiti con inibizioni e sensi di colpa, sono repressi in nome del sacrificio e del martirio; tanto da fare di questi i due leitmotive della sua vita. L'amore paterno, l'attrazione fisica per Sara, i propri beni materiali, la propria posizione sociale e perfino la propria dignità, tutto viene sacrificato, cioè represso, in nome di un bene futuro, non di questa terra, che la sua fede gli fa pensare come un dovuto compenso per tutte le tribolazioni e le sofferenze patite. In altri termini, Diego si autopunisce al fine di ottenere una ricompensa ben superiore ed eterna.

In questo rapporto di do ut des con il Dio della sua fede, Diego non sembra rendersi conto del fatto che è lui, uomo, a definire la divinità a propria immagine e somiglianza e ad imporle le proprie categorie del bene e del male e di come queste debbano essere applicate e ricompensate nell'aldilà. Così facendo prepara la via al vuoto ed al nulla che è destinato a scoprire quando tutto il suo solido ma illusorio edificio di certezze astratte crollerà miseramente, perché costruito su un autentico vuoto di fede. La fede, che è prima di tutto slancio coraggioso verso l'ignoto, è stata imbrigliata in rigide categorie mentali, è stata razionalizzata ed ha perso la sua capacità espansiva, la sua carica di energia vitale, come simbolicamente suggeriscono il cipresso ed il crocefisso che dal muro di cinta connotano la casa ed il suo padrone: il cipresso, che 'altissimo' e severo si proietta verso il cielo, è il simbolo di una spiritualità che, tutta volta verso Dio, nulla concede alla terra su cui cresce; la croce, simbolo di sofferenza per antonomasia, che si staglia 'grande' e 'nera', e il Cristo 'squallido' e 'sanguinante' come il sacrificio di Diego, sono il corrispettivo simbolico della sua condizione spirituale. Diego come il Lazzaro biblico è irrigidito nella morte e solo il miracolo di una fede vivificante potrà farlo rinascere a nuova vita. Sotto questo aspetto il parallelo col personaggio biblico coincide perfettamente, ma Pirandello nel suo dramma esplora il 'dopo' miracolo, la problematica che nasce nel personaggio dopo la resurrezione. Il ritorno in vita di Diego grazie alle arti mediche del dottor Gionni è in effetti solo un pretesto per indagare ben altro: l'aldilà, l'Oltre della vita e della morte, la questione che l'uomo da sempre si è posto davanti al mistero dell'esistenza umana. Questo aldilà per Diego Spina si è rivelato il Nulla assoluto, tanto da non averne neppure memoria. Il suo Dio, creato a costo di tanto sa- 
crificio e martirio, non esiste. Il vuoto delle sue astrazioni gli si manifesta infine, come si legge nella didascalia finale del secondo atto:

Diego Spina è bianco di terrore, con gli occhi sbarrati nel vuoto. Tutti lo guardano sospesi e smarriti, seguitando a tenere il silenzio, che è quello esterrefatto della vita davanti alla morte. (1209)

Se c'è solo il nulla, allora i pilastri della sua intera vita (fede, sacrificio, martirio) crollano miseramente, come sottolinea Pirandello stesso nell'intervista rilasciata a Cavicchioli:

Nel Lazzaro do la risposta più netta al dissidio fondamentale, nel mio teatro, in quanto fatto religioso e sociale. Se all'uomo non libero togliete la forma, in quanto legame spirituale, subito egli ricasca fra le bestie, e il primo atto della sua così detta libertà è una fucilata contro un altro uomo, contro l'Adamo nuovo che vive in pace con la sua Eva. (22)

Sara, l'Anima in senso junghiano, cioè il carattere dinamico e trasformatore del Femminino, farà da tramite tra questo stato di squallida spiritualità e il suo possibile rinnovarsi in qualcosa di vitale e vibrante, che finalmente integri il femminile nel maschile, l'inconscio nel conscio, il mortale nell'immortale, e accetti Dio ed il suo mistero per quello che sono e senza patti.

In questo senso l' "apparizione" numinosa di Sara, la Donna-Natura, la novella Eva, nel tramonto infuocato del primo atto ha tutte le caratteristiche della messaggera di morte e di vita, come il nero ed il rosso di cui è vestita. La sua venuta porterà non solo al primo grave crollo delle illusioni di Diego l'avvenuto abbandono degli ordini religiosi da parte del figlio — , ma anche alla sua stessa morte fisica. Nel contempo, però, ne preparerà anche la futura rinascita non tanto del corpo quanto dello spirito. Prima, però, la sua disperazione deve toccare il fondo ed i suoi mostri interiori, troppo a lungo repressi, devono manifestarsi in tutta la loro violenza. Nella fucilata ad Arcadipane trovano sfogo infine l'onta, la gelosia, quel "bottone di fuoco" che era sempre rimasto acceso:

Tenermi; non far nulla - così - vivere del mio strazio; lasciarlo durare, senz'offrirgli il più piccolo sfogo, anzi, come un bottone di fuoco, lo scherno della gente, fu la mia vittoria: il martirio. Lungo. Lungo, perché la ferita si riapriva sempre, e il sangue - sangue cattivo - tornava a sgorgare. (1177)

Nel dare sfogo alla belva che ha dentro di sé, nell'integrare finalmente i propri istinti e gli impulsi più sottaciuti nella coscienza - tutti accentrati intorno alla figura di Sara - Diego compie il primo passo verso una nuova condizione di vita.

Le ultime due battute di Diego sono la spia di un importante passo sulla via della trasformazione. La prima ("Ho voluto ... ho voluto uccidere ... . e tutto il male che ho fatto ..." 1222) è l'ammissione per la prima volta delle sue colpe, dopo che ostinatamente aveva continuato a negarlo mascherandosi dietro i suoi principi; è il guardarsi dentro, finalmente libero da schemi preconcetti e da desi- 
deri inammissibili; è il trovarsi faccia a faccia con i propri errori e le proprie colpe ed accettarne la responsabilità. La seconda battuta ("Che debbo fare? che debbo fare?" 1222) indica l'accettazione di un cambiamento che dovrà avvenire nella sua vita e la ricerca di quale esso debba essere, con lo stesso sentimento di pietrificato stupore che doveva aver invaso il Lazzaro biblico quando riaprì gli occhi alla vita.

La vera rinascita di Diego non è stata quella fisica ad opera del medico, ma quella pienamente spirituale che gli si apre davanti. Paradossalmente per Diego Spina la resurrezione non tanto fisica ma spirituale potrà avvenire proprio tramite quella 'materia', la base terrena della realtà, che egli aveva sempre negato e represso. L'iniezione al cuore (l'organo per eccellenza dei sentimenti e degli affetti), che lo ha riportato in vita, diventa così emblematica della necessità di ridare vita ad una funzione che era stata negata, pietrificata e la fucilata ad Arcadipane è il primo segno, anche se di tipo distruttivo, che la funzione del sentire è stata riattivata. È nel ritrovare il contatto con l'Anima, intesa junghianamente come archetipo della vita e dell'interiorità, che a Diego si schiude la via verso la rivelazione del divino nell'umano, come spiega Pirandello nell'intervista di Drioli: "Il mondo dell'al di là [. . .] si crea necessariamente perché gli uomini che non arrivano a scoprire Dio in se stessi, hanno bisogno di crederlo presente nell'al di là". E a questa rivelazione del divino in se stessi sarà indispensabile il contributo di Lucio.

Prima però di proseguire con quest'altro personaggio, conviene soffermarsi ancora brevemente sul rapporto tra Io ed Anima in Diego Spina, cominciando da un'osservazione di Neumann (Storia 329): "Mentre l'uomo comune non ha un'anima propriamente sua, perché è il gruppo, col suo canone di valori, che gli prescrive ciò che egli può o non può essere psichicamente, l'eroe è colui che possiede una sua anima perché l'ha conquistata lottando". Diego Spina, accettando pienamente i valori del gruppo è stato finora un "uomo comune", senza qualità eroiche, ma solo con velleità. Il suo sacrificio è stato sterile e invece di rinnovamento ha soltanto portato morte e distruzione. Si è definita la sua condizione sopra come il risultato di una castrazione patriarcale, in cui cioe il sistema conscio è sopraffatto dallo spirito e perde la necessaria compensazione da parte dell'inconscio. Si riconosce, dunque, in Diego Spina il ritratto di quella che Neumann (Storia 335) chiama "inflazione dello spirito" e considera essere "il limite estremo di un tipico sviluppo occidentale". Se si tratta dunque di un aspetto dello sviluppo psichico occidentale che si può considerare tipico, sembrerebbe abbastanza giustificabile allora intravedere nel ritratto di Diego Spina fattone da Pirandello e nel grave rischio di totale disastro della sua vita un monito ed un tentativo di additare un modello di comportamento e di pensiero più desiderabile per l'uomo contemporaneo. Per questo Pirandello ci ha tenuto a precisare che il suo "è un Lazzaro di oggi". 9

Alla fine dell'opera pirandelliana, e solo allora, con il crollo delle illusioni della coscienza e tramite una nuova e sofferta apertura verso la parte più istin- 
tuale ed emotiva di sé, Diego incontra la sua Anima e per la prima volta la riconosce. Se da eroe la libererà dal drago patriarcale è una questione che viene lasciata aperta, ma che consente già, tuttavia, di riconoscere nel personaggio di Diego in embrione la figura archetipica dell'eroe che combatte per liberare la principessa prigioniera del drago paterno. Spiega ancora Neumann (Storia 183, 190) a proposito di questo archetipo: "La prigioniera liberata non è solo il simbolo del rapporto erotico puro e semplice col femminile; l'azione eroica deve liberare, con la prigioniera, anche il rapporto vivo con il Tu, con il mondo in generale. [. . .] Dall'unione del lato creativo dell'anima con la coscienza egoica dell'eroe, che ora è capace di conoscere il mondo, di dargli forma e di agire in esso, da quest'unione deriva la vera nascita, che è la sintesi dei due aspetti". In questa unione è da rintracciare il significato più profondo del mito pirandelliano che nel personaggio di Diego presenta solo una delle sue molteplici sfaccettature; le altre, che compaiono soprattutto nei personaggi di Sara e di Lucio, contribuiscono a svilupparlo nella sua globalità.

Nel personaggio di Diego s'intravede, quindi, un inizio di inversione del motto patriarcale "via dal mondo materno, verso il mondo paterno", e nella sua figura iniziale di Antiadamo - non è un caso infatti che spari ad Arcadipane/Adamo - , è contenuto tuttavia il germe della sua possibile trasformazione verso la totalità psichica di conscio ed inconscio, di Io e di Anima, processo che invece compirà il suo intero corso in Lucio e che però non è possibile discutere interamente in questa sede per ovvie ragioni di spazio.

\section{La ribellione del Figlio}

Il personaggio di Lucio rappresenta nel Lazzaro la figura perfettamente compiuta - insolita nelle opere pirandelliane anche del periodo mitico - dell'eroe archetipico. ${ }^{10}$ Di tale figura, tuttavia, è di necessità considerare ora soltanto l'aspetto più strettamente attinente al discorso sulla riscrittura del mito biblico.

Al centro della vicenda di Lucio compare la figura del padre: è il padre che l'ha staccato forzatamente ed innaturalmente ancora bambino dalla madre, è il padre che lo ha indirizzato verso la carriera ecclesiastica, trasmettendogli i propri valori culturali e religiosi. Ma proprio perché l'esito di tutte le sue iniziative è l'infelicità e la mancata realizzazione del figlio come individuo, questa figura paterna assume le chiare sembianze del Maschile Terribile archetipico, che impedisce al figlio di divenire se stesso. A proposito di questo versante dell'archetipo del Padre precisa Neumann (Storia 171): "Egli opera come un sistema spirituale che imprigiona e uccide la coscienza del figlio [. . . . Questo sistema spirituale si incarna nella forza vincolante della vecchia legge, della vecchia religione, della vecchia etica, del vecchio ordine sociale; come coscienza morale, come convenzione, come tradizione o come un qualunque altro fenomeno spirituale che blocca il figlio e gli impedisce di accedere al futuro". Si tratta, cioè, della medesima condizione di castrazione patriarcale che Diego, anch'egli vittima ma senza averne coscienza, ha a sua volta perpetrato sul suo stesso figlio. 
La situazione psicologica iniziale di Lucio è, quindi, quella di Isacco, potenziale vittima sacrificale che il padre è pronto a sacrificare al divino. ${ }^{11}$ È chiaro, tuttavia, fin dalle prime battute del dramma che Lucio ha introdotto nel modello biblico di Abramo e Isacco un elemento nuovo: ha rifiutato il proprio ruolo di vittima, si è ribellato al padre castrante, abbandonando il sacerdozio. Questa azione, che lo ha trasformato da vittima passiva in eroe, lo pone pertanto in una precisa linea di sviluppo in senso archetipico. L'eroe, infatti, che è tale proprio perché e in quanto si oppone all'ordine vecchio per portarne uno nuovo, ${ }^{12}$ per affermarsi come tale deve affrontare il padre e ucciderlo. Puntualmente questo evento ha luogo nel dramma: Diego, come diretta conseguenza dell'azione di Lucio, di cui si è fatta messaggera Sara, perde la testa e viene ucciso da una macchina per strada. La morte di Diego, quindi, è l'occasione per un'esplorazione del tema della rinascita non solo in termini strettamente biblici ma anche nelle sue più vaste $\mathrm{e}$ profonde implicazioni archetipiche.

Il titolo dell'opera ed i suoi episodi centrali indicano chiaramente la resurrezione come il suo tema di fondo, come si è visto. Ciò che, però, non è ovvio a prima vista è la riscrittura del mito biblico. Alla base del mito della rinascita Pirandello, infatti, pone il problema morale ed esistenziale di Abramo e di Isacco, l'uno di fronte al suo Dio e l'altro al proprio padre. Come Abramo obbedisce ciecamente alla richiesta divina di sacrificare l'unico figlio, così Diego, l'Abramo in veste moderna, segue alla lettera la legge del suo credo che porta anche lui a sacrificare il proprio figlio. La sua cieca obbedienza viene scossa drammaticamente, tuttavia, dal rifiuto del figlio di essere sacrificato. Il personaggio di Lucio/Isacco, quindi, è di fondamentale importanza nell'economia dell'opera, perché è il portatore del messaggio di disobbedienza, che diventa così il fulcro della riscrittura mitica pirandelliana. Andando contro il principio di autorità patriarcale, che dal padre terreno si estende verticalmente fino a quello divino, l'Isacco moderno afferma un nuovo modo di vivere la fede e la vita stessa, apportandovi il senso di fiducia che l'uomo possa essere capace di contenere in sé il divino senza doverlo cercare in un fantomatico altrove.

Con la sua azione di rottura e di ribellione, inoltre, l'Isacco moderno cessa di essere la vittima del principio superiore e si pone, invece, lui stesso, in quanto eroe, come portatore di un nuovo principio spirituale. Con la trasformazione di Lucio/Isacco da passiva vittima sacrificale in eroe attivamente impegnato a dare senso e scopo alla propria vita, Pirandello aggiunge e sviluppa una nuova, fondamentale dimensione del mito biblico, secondo il grande modello archetipico dell'eroe alla ricerca del tesoro. Il tesoro, che porta alla rinascita tramite la scoperta del Sé, ha quindi come condizione indispensabile per ottenerlo la disobbedienza.

È a causa della sua disobbedienza che Eva perde il paradiso e conosce il dolore, ma è anche e solo grazie al rifiuto di uniformarsi ai ruoli di madre e di moglie quali le vengono imposti dalla società, che la moderna Eva/Sara riacquista il suo Eden e con esso la propria interezza e pienezza di individuo. Alla base 
del principio di disobbedienza che Pirandello pone come conditio sine qua non al processo di rinascita si stabilisce, quindi, anche ciò che lo qualifica: la disobbedienza è nei confronti del vecchio, del superato, dell'immobile, di tutto ciò che in altri termini blocca la vita cercando di fermare il suo perenne ed inarrestabile fluire in forme cristallizzate. L'aspetto positivo della disobbedienza è l'accettazione del continuo cambiamento e rinnovamento come parte della sfida della vita stessa.

La figura dell'eroe, che in quest'opera pirandelliana presenta principalmente due delle sfaccettature di un unico grande archetipo - la ricerca del tesoro (Lucio) e la liberazione della principessa prigioniera/Anima (Diego) - si fa portante di tale qualità dinamica di costante rinnovamento e di conquista di sé. La costellazione dell'archetipo dell'eroe nella psiche instaura un processo di dilatamento della coscienza, tramite il recupero dell'inconscio, la Grande Madre e l'Anima della psiche dell'uomo, svalutata e repressa nel mondo moderno. Grazie all'integrazione dell'Eros nel Logos, come traspare dalle parole di Monsignor Lelli davanti alla trasfigurazione di Lucio: "Figlio mio benedetto, ecco che Dio dalla mente ti ridiscende nel cuore!" (1216), è possibile ri-indirizzare e compensare l'unilateralità della coscienza moderna, troppo a lungo e troppo a fondo dominata dai valori astratti e oppressivi della mentalità patriarcale. La fede che, appunto, si trasferisce dal mondo astratto delle idee a quello caldo e vivificante della carità, è viva e vitale perché partecipa del pieno delle energie più autentiche e profonde dell'essere. Non è più dogma o precetto, ma vita. Il discorso pirandelliano nel Lazzaro, anche se tratta un tema religioso, finisce per acquistare, quindi, ben più ampio respiro, investendo del suo messaggio il senso della vita intera.

Quando Pirandello chiama il suo dramma "mito" e nel contempo lo colloca nel "tempo presente" stabilisce un voluto contrasto. Il mito per l'uomo moderno è spesso nient'altro che una favola curiosa inventata tanto tempo fa da uomini che erano come bambini; è difficile per lui vederne coscientemente il sotteso legame con la sua condizione presente. Il senso della riscrittura pirandelliana, pertanto, sembra essere quello di recuperare il mito e trasformarlo, così da renderlo emblematico e significativo anche per l'uomo d'oggi. L'intento dichiaratamente didattico del suo mito risponde alla volontà dell'autore di andare incontro ai bisogni dell'uomo. L'azione di carità che Lucio compie a conclusione del dramma sacrificando la propria vita al servizio degli altri, trova il suo corrispettivo, in un certo senso, in quella dell'autore stesso di accantonare lo scetticismo di una intera vita di scrittore per "creare il terreno sotto i piedi" di chi cammina sull'abisso (in Giudice 496-97).

Pirandello nel Lazzaro ha quindi attinto alle fonti bibliche per il suo mito moderno, spesso fondendole - come s'è notato - in una fitta rete di rapporti con elementi mitici pagani. Tali recuperi mitici, siano essi giudaico-cristiani o greci, sono stati traslati in chiave moderna. Che questo processo di traslazione sia stato possibile non sorprende, in quanto nei miti del passato si manifesta quello stesso materiale inconscio collettivo che, comune all'uomo moderno come a 
quello antico, viene distillato sotto forma di archetipi di tutta l'Umanità. Ciò che interessa notare, semmai, - e qui sta il merito dell'opera pirandelliana - è il modo in cui tali archetipi sono stati, per così dire, rielaborati contribuendo, di conseguenza, a dilatare e/o ri-indirizzare la coscienza collettiva dei suoi contemporanei verso un recupero della totalità dell'uomo e della vita, che nasce dall'attingere direttamente alle sue fonti primigenie.

La scelta mitica nel Pirandello maturo nasce dalla profonda consapevolezza della crisi, sofferta in prima persona, dell'uomo contemporaneo e dall'ansia di totalità che ne deriva. Nel Lazzaro vengono esplorati il mistero stesso ed il senso ultimo della vita e della morte e la conclusione del dramma non sembra lasciar dubbi su quale venga privilegiato dei due modelli di esplorazione proposti nel dramma, uno che cerca le sue risposte in un remoto 'altrove' e l'altro che invece le trova nelle radici stesse dell'essere umano.

In un'opera di dieci anni prima (Si gira, 1916) Pirandello aveva scritto: "C'è un oltre in tutto. Voi non volete o non sapete vederlo ...". ${ }^{13}$ Che cos'è questo oltre? Le "misteriose illusioni" di cui Pirandello parla in Non si sa come? ${ }^{14} \mathrm{Pi}$ randello non cerca neppure lontanamente di dare una risposta razionale e finale al quesito che da sempre ha assillato l'umanità; il suo discorso poetico s'incentra piuttosto sulla fede, intesa non come religione istituzionalizzata ma come atteggiamento interiore. Da questo punto di vista Lazzaro, come del resto anche gli altri due miti pirandelliani, sono una ricerca di fede senza infingimenti né sentimentalismi; sono una ricerca dei valori assoluti che sottendono il mistero della vita.

È stato notato come nella sua opera Pirandello presenti costantemente una visione labirintica dell'esistenza e giunga a considerare il suo stesso essere scrittore un labirinto senza uscita, come per il protagonista di Quando si è qualcuno (Sicari; Cipolla 114-31). Lazzaro potrebbe fornire, allora, il filo di Arianna per uscire da una forma irrigidita e da un labirinto altrimenti senza uscita? La risposta sembra darla Anselmo Paleari:

[... ] non possiamo comprendere la vita, se noi in qualche modo non ci spieghiamo la morte! Il criterio direttivo delle nostre azioni, il filo per uscire da questo labirinto, il lume, insomma, signor Meis, il lume dovrà venirci di là, dalla morte. Col buio che ci fa? Buio? Buio per Lei! Provi ad accendervi una lampadina di fede con l'olio dell'anima. Se questa lampadina manca, noi ci aggiriamo qua, nella vita, come tanti ciechi con tutta la luce elettrica che abbiamo inventato". (Tutti i romanzi 121)

Lucio, nome emblematico, in Lazzaro ha proprio fatto questo: ha "acceso una lampadina di fede con l'olio dell'anima".

University of South Africa

NOTE

1 Pirandello parlerà diffusamente di entrambi i miti, infatti, nell'intervista rilasciata a Romano Drioli per la Gazzella del popolo nel giugno del '26, e sul Lazzaro in particolare si 
esprime come se l'opera fosse già stata compiutamente scritta: "Ho scritto infatti un Lazzaro. [. . .] un Lazzaro moderno . . . un'opera di tale impostazione [. . .] definitivamente chiarirà il mio punto di vista sul mito religioso. [. . . ] Il mio nuovo dramma crea il mito religioso e affronta il problema dell'immanenza".

2 Altri lavori pirandelliani che raggiunsero le scene straniere prima di quelle italiane sono Diana e la Tuda data in versione tedesca a Zurigo nel 1926, Questa sera si recita a soggetto presentata anch'essa in versione tedesca a Koenigsberg nel gennaio del '30, Sogno (ma forse no) e Quando si è qualcuno le cui prime mondiali ebbero luogo rispettivamente a Lisbona nel '31 e a Buenos Aires nel '33. Infine la prima rappresentazione di Non si sa come, l'ultima opera compiuta di Pirandello, avvenne al Teatro Nazionale di Parigi nel dicembre del 1934 subito dopo la presentazione del Premio Nobel all'autore.

3 Il Sé è per Carl Gustav Jung, di cui si segue qui il pensiero, il punto d'arrivo dell'esistenza individuale, è l'eterno, il divino che è in ciascuno di noi e, in quanto tale, rappresenta il senso finale del destino individuale come parte del Tutto. Il Sé è il punto d'incontro e di fusione degli opposti e delle dicotomie (bene/male, luce/ombra, amore/odio, sacrificio/egoismo, vita/morte, ecc.), che lacerano il tessuto della vita. Sul piano psicologico il Sé rappresenta l'integrazione del materiale archetipico dell'inconscio collettivo nella coscienza individuale e, come tale, rappresenta il culmine del processo d'individuazione. Per una trattazione completa dell'archetipo del Sé, cfr. Jung.

4 Cfr. Neumann a proposito dello sviluppo della personalità: "Lo sviluppo della coscienza egoica procede parallelamente a una tendenza a rendersi indipendente dal corpo. Tale tendenza è molto evidente nell'ascesi maschile, nemica della terra, del corpo e della donna [. . .]. Nell'indipendenza dal corpo, nella vittoria sul dolore, sulla paura e sulla sessualità che provengono appunto dal corpo e cercano di sopraffare l'Io, in queste esperienze elementari l'Io sperimenta la propria maschilità superiore" (Storia 272).

5 Cfr. Neumann: "L'altra forma della castrazione patriarcale è l'identificazione con il padre divino. È lo stato di possessione prodotto dall'inflazione del cielo, «l'annullamento mediante lo spirito». [. . .] Mentre la castrazione matriarcale ha un orientamento orgiastico, quella patriarcale ha un orientamento ascetico" (Storia 171-72).

6 Riassumendo il significato dello hieròs gámos nella visione di Neumann, la Pulcini precisa che "tramite la coniunctio con la coscienza apollinea maschile [la sapienza peculiarmente femminile] permette di tornare a celebrare, in una forma più cosciente ed individuata, l'antico hieròs gámos tra il sole e la luna, tra il maschile e il femminile, costellando la totalità del Sé" (189).

7 A proposito del carattere sacro e del valore simbolico del pane, cfr. Neumann: "Il simbolismo della trasformazione diviene sacrale ovunque alla natura di pura trasformazione del processo faccia seguito l'intervento umano, che eleva la trasformazione naturale, così che in questo processo risultino attivi non solo la natura o l'inconscio, ma anche la personalità umana, che attraverso la sua attività viene sempre compresa nel processo di trasformazione" (La Grande Madre 66).

8 L'Anima come archetipo della vita, in senso junghiano, non è un'entità spirituale ma psichica e rappresenta l'elemento femminile - non in senso letterale sessuale ma simbolico - nella psiche inconscia maschile (il suo corrispettivo nella donna è l'Animus); è quindi una figura che compensa la coscienza maschile e nella sua funzione più alta "rappresenta quella forma di saggezza spirituale che scaturisce dalle fonti primarie ed inconsce dell'energia" (Neumann, La Grande Madre 322). Essa è, inoltre, "la sapienza peculiarmente femminile [Sophia] che [. . .] può contenere e ricevere e [. . .] può allo stesso tempo, illuminare e trasformare" (Pulcini 189).

9 Cfr. Nota introduttiva alla prima edizione di Lazzaro, vol. 26 delle Maschere nude. Milano: Mondadori, 1929.

10 Per una trattazione, anche se sommaria, di tale aspetto dell'opera pirandelliana rimando al mio saggio, "Il mito di Lazzaro", mentre sull'evoluzione della poetica pirandelliana nella 
fase mitica cfr. l'altro mio saggio, "Arte e mito".

11 Una riprova, se mai occorresse, del recupero consapevole del modello biblico di Abramo e Isacco da parte di Pirandello per i propri personaggi è data dal nome di Sara che è, com'è noto, moglie e madre rispettivamente di Abramo ed Isacco ed anche - certo non a caso - di Diego e di Lucio.

12 Cfr. Neumann: "Proprio le persecuzioni e i pericoli che la figura del re o del padre ostile impone all'eroe lo rendono tale. Gli ostacoli che il vecchio sistema paterno cerca di opporre costituiscono perciò la condizione indispensabile per l'azione eroica. [. . .] Essi costituiscono un canone necessario di eventi che contraddistinguono sia simbolicamente che obiettivamente la natura dell'eroe, il quale come portatore del nuovo deve uccidere l'antico" (Storia 161-62).

13 Ora col titolo Quaderni di Serafino Gubbio operatore in Tutti i romanzi 1109.

14 In Maschere nude 838. Idee che sono poi riprese anche nella novella "Di sera, un geranio" (1934), in Novelle per un anno 2.814.

\section{OPERE CITATE}

Cavicchioli, Giovanni. "Introduzione a Pirandello". Termini 1.2 (ottobre 1936): 22-23.

Cipolla, Gaetano. Labyrinth. Studies on an Archetype. New York: Legas 1987.

Drioli, Romano. "La nuova colonia e Lazzaro: i nuovi lavori di Pirandello nel pensiero dell'autore". Gazzetta del popolo. 12 giugno 1926.

Durand, Gilbert. Le strutture antropologiche dell' immaginario. Introduzione all'archetipologia generale. Trad. Ettore Catalano. Bari: Edizioni Dedalo, 1984.

Giudice, Gaspare. Luigi Pirandello. Torino: Utet, 1963.

Jung, Carl Gustav. Aion: ricerche sul simbolismo del Sé. Vol. 9, tomo 2. Opere complete. Trad. Lisa Baruffi. Torino: Bollati Boringhieri, 1982.

Massi, Lucia. "Pirandello's Theory of Modern Myths". The Yearbook of the British Pirandello Society 6 ( 1986): 1-18.

Meda, Anna. "Il mito di Lazzaro come esplorazione dell'Oltre in chiave archetipica". Pirandello e l'Oltre. Ed. Enzo Lauretta. Milano: Mursia, 1991. 289-301.

. "Arte e mito nell'ultimo Pirandello". Studi d' italianistica nell' Africa australe 4.2 (1991): 19-42.

Neumann, Erich. Storia delle origini della coscienza. Trad. Livio Agresti. Roma: Casa Editrice Astrolabio, 1978.

La Grande Madre. Fenomenologia delle configurazioni femminili dell' inconscio.

Trad. Antonio Vitolo. Roma: Casa Editrice Astrolabio, 1981.

Pirandello, Luigi. Lazzaro. In Maschere nude. Vol. 2. Milano: Mondadori, 1962. 1059-158.

. Tutti i romanzi. Ed. Corrado Alvaro. Milano: Mondadori, 1963.

Novelle per un anno. Voll. 1-2. Ed. Corrado Alvaro. Milano: Mondadori, 1964.

Pulcini, Elena. "L'eros e la coscienza lunare". Aut Aut 237-38 (maggio-agosto 1989): 79-91.

Sicari, Carmela. Lo specchio e lo stigma. Il racconto pirandelliano. Ravenna: Longo, 1979. 